\title{
Clinical Evaluation of the GENIUM AFI 5.5 Prepak in Cataract Surgery
}

\author{
a report by \\ Patrice Vincent
}

LCA Pharmaceutical Clinical Research

DOI: $10.17925 / E O R .2009 .02 .01 .25$

The demand for intraocular lenses (IOLs) pre-loaded in single-use sterile injectors is a logical step in the evolution of cataract surgery. The intention is not only to dispense with the surgeon or his/her assistant loading the IOL in a conventional injector, but also to directly implant the IOL inside the eye correctly and safely, reducing contact with the environment to virtually zero, and ultimately to make surgery easier and safer, preventing endophthalmitis.

The obvious advantage of such a technique is the elimination of risks such as mistakes during $\mathrm{IOL}$ handling (incorrect placement inside the cartridge, accidental contamination, etc.) or even the use of an improper injector device, resulting in a damaged lens. The latter point demonstrates that there is a big difference between a single-use injector that has to be loaded conventionally and a truly pre-loaded IOL. To fulfil this demand, LCA Pharmaceutical has designed the GENIUM AFI 5.5 Prepak, an innovative truly pre-loaded hydrophilic IOL (see Figure 1).

\section{Clinical Evaluation of the GENIUM AFI 5.5. Prepak}

\section{Methods - Study Design and Subjects}

A European multicentre open clinical study was designed with the primary objective of assessing the performance of the GENIUM AFI 5.5 Prepak in terms of visual acuity improvement, and then to determine any potential adverse effects peri- and post-operatively. Overall therapeutic efficacy was also evaluated (assessment by investigator). The study was conducted between May 2004 (first inclusion) and June 2005 (latest results). The study protocol was based on ISO 13503-7, including three reports (pre-, peri- and post-operative at $2 \pm 1$ months). Ten investigators were selected, each treating 30 patients suffering from senile cataracts. Three hundred and one patients were treated (one investigator treated 31 patients).

The inclusion criteria were age greater than 50 years (both sexes) and scheduled extraction of the crystalline lens by phacoemulsification followed by implantation in the capsular bag of a GENIUM AFI 5.5 Prepak pre-loaded IOL. The exclusion criteria were traumatic cataract, uveitis, keratitis or other

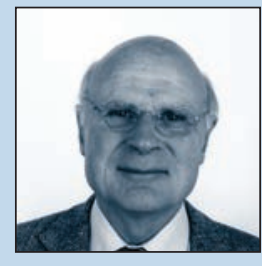

Patrice Vincent is a founder member and Research and Development Manager of LCA, a French company that develops and manufactures medical devices including intraocular lenses (IOLs), ophthalmic viscosurgical devices, single-use sterile surgical instrumentation and single-dose eye solutions. He developed the GENIUM single-use preloaded IOL injector from 1999 to 2004. His background includes mechanics, structural engineering, rheology, precision machinery and optics.
Figure 1: GENIUM AFI 5.5 Prepak Pre-loaded Intraocular Lens with Injector in Immersion Inside Its Sealed Sterile Vial

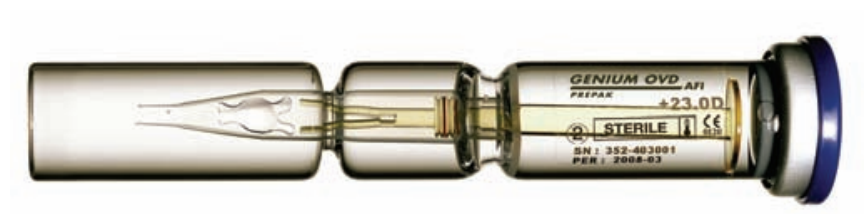

The vial itself is delivered inside a sealed pouch for double sterility protection.

inflammation of the anterior segment, uncontrolled glaucoma and any retinopathy that would prevent satisfactory visual acuity being obtained.

The patient group ranged from 46 to 99 years of age (mean age $75.2 \pm 8.1$ years) and $64 \%$ were women. Age distribution is shown in Figure 2. Very few deviations to the protocol were accepted: three patients with early senile cataracts and 13 patients with posterior segment diseases who achieved visual improvement were included. The GENIUM AFI 5.5 Prepak was implanted in all patients. The whole range of powers (+14.0 to $+26.0 \mathrm{D})$ available at the time of the study were used. Distribution of these powers is shown in Figure 3.

\section{Device Description}

GENIUM AFI 5.5 Prepak is a foldable acrylic hydrophilic IOL pre-loaded in a single-use injector. The whole system is delivered sterile, double-packed in a sealed glass vial containing a solution. Sterility is obtained by steam autoclaving. The GENIUM injector and the amniotic fluid index (AFI) IOL have been specifically designed together for an easy and reproducible injection. The GENIUM injector and IOL have several unique features:

- The GENIUM injector is simple, being made from two major components - syringe and piston - and extremely compact.

- The syringe, or 'injector cartridge', containing the unfolded IOL is made of transparent polypropylene, allowing full visual control at any stage of folding and injection. The bevelled and flattened tip is designed to go through a $3.2 \mathrm{~mm}$ incision without enlarging it.

- The patented 'folding piston' has two exclusive flexible pushing rods to maintain the $\mathrm{IOL}$ in perfect alignment during folding and injection. As it is made of polyetherimide it is strong, stable and heat-resistant, and its natural amber colour contributes to the ease of visual control.

- No separate IOL container is used and no mechanism is required to compress the IOL prior to pushing the piston.

- The lubricating viscoelastic solution (ophthalmic viscosurgical device [OVD]) can be easily introduced from the injector tip using a standard cannula.

- A bimanual technique is recommended when handling the GENIUM injector as it offers better control. Also, a retraction of the piston is 
Figure 2: Patient Age Distribution $(n=301)$

GENIUM AFI 5.5

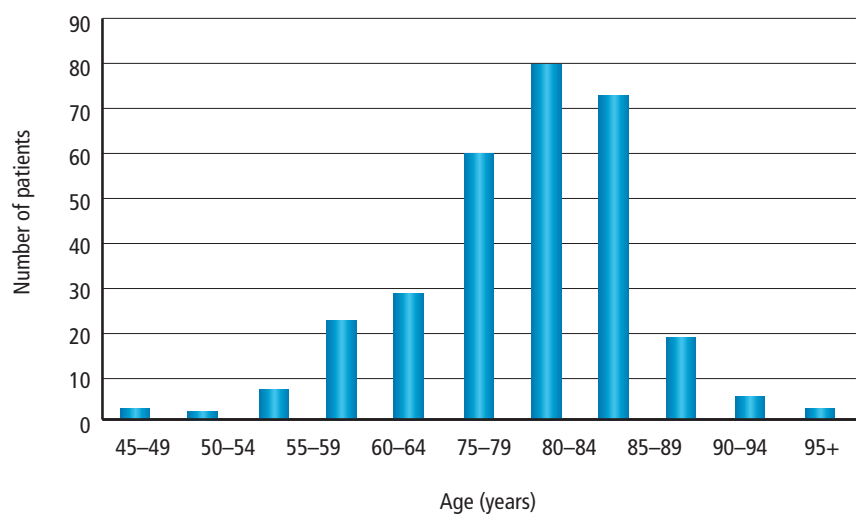

Figure 3: Intraocular Lens Power Distribution $(n=301)$

GENIUM AFI 5.5

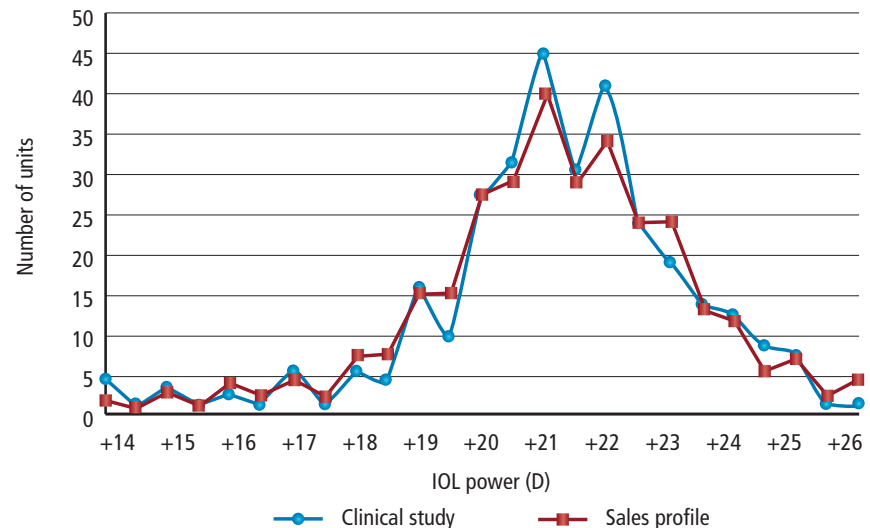

Power distribution is identical between the intraocular lens (IOL) used for the study and IOLs sold during the same period - here at proportional scale - confirming that the population sample studied is representative.

Figure 4: Visual Acuity Distribution $(n=291)$

GENIUM AFI 5.5

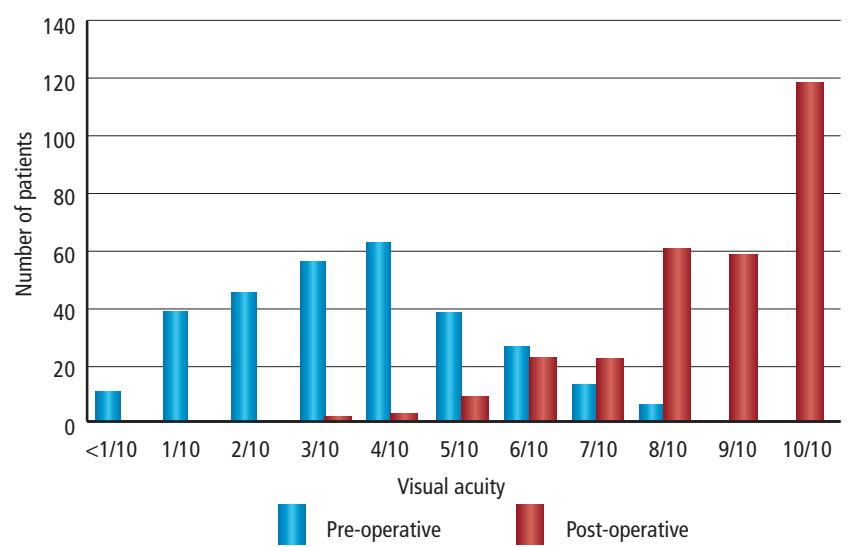

required to release the $\mathrm{IOL}$; this allows the $\mathrm{IOL}$ to be directly injected in a horizontal position and placed directly in the capsular bag, without needing to use another instrument.

- The patented AFI tripod IOL is specifically designed to fit the GENIUM injector. The two superior haptics in contact with the rods keep the $\mathrm{IOL}$ aligned until the optic is folded; the narrow single inferior haptic can precede the optic during injection.
Figure 5: A-constant Distribution $(n=280)$

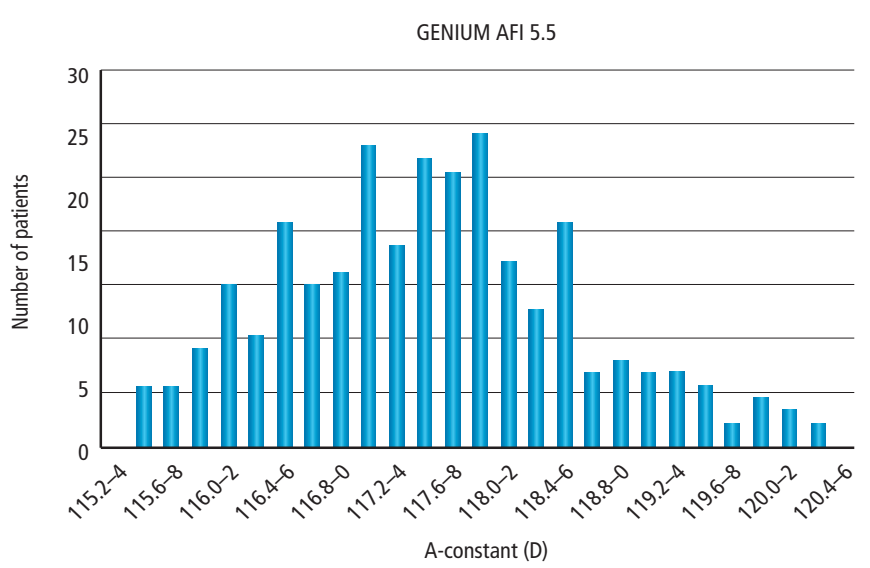

Figure 6: Intraocular Pressure - Follow-up $(n=294)$

GENIUM AFI 5.5

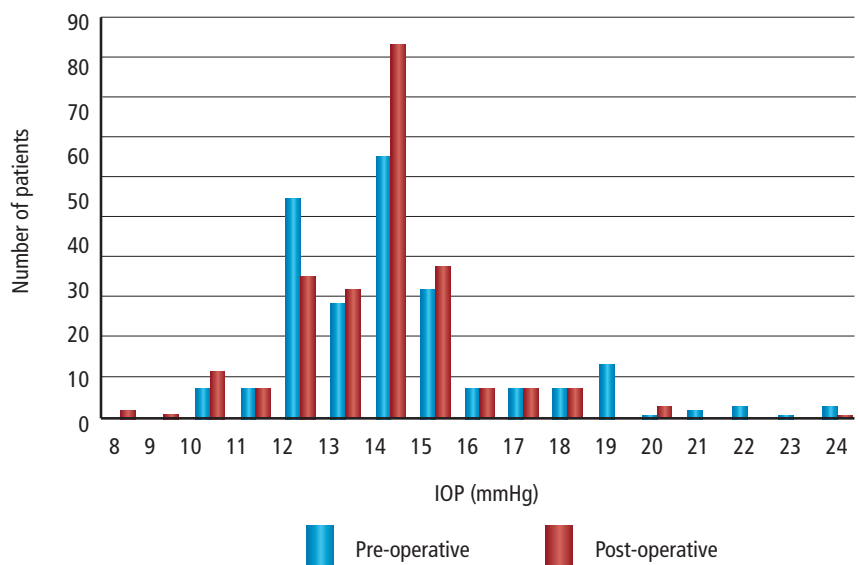

- The AFI tripod IOL is easy to place and to centre, offering great stability inside the capsular bag.

\section{Results}

Peri-operative Results

Peri-operative results are shown in Table 1.

\section{Post-operative Results}

Quantitative results comparing pre- and post-operative outcomes are shown in Table 2. Keratometry changes were also analysed: $85 \%$ of corneas remained unchanged at $\pm 0.25 \mathrm{D}$, indicating that $3.2 \mathrm{~mm}$ incisions with the GENIUM AFI 5.5 Prepak do not induce astigmatism. Visual acuity (VA) recovers well with the GENIUM AFI 5.5 (see Figure 4). Of 47 patients with a pre-operative VA of $0.1(1 / 10=20 / 200)$ or less, 41 recovered $0.8(8 / 10=20 / 25)$ and none recovered less than 0.5 $(5 / 10=20 / 40)$. The patients with a final VA of $0.4(4 / 10=20 / 50)$ or less had corneal problems or demonstrated poor co-operation, but their VA was always improved. Comparison of refractive correction with the refractive target shows a significant residual myopia of around $-0.50 \mathrm{D}$ (sphere). Therefore, the A-constant is obviously oversized at $118.3 \mathrm{D}$. As calculations performed by investigators are ignored, a statistical approach was made using the SRK II formula. The A-constant, re-calculated from SRK II, shows a relatively wide distribution (see Figure 5). However, this distribution is narrower for several individual 
Table 1: Peri-operative Results with the GENIUM AFI 5.5 Prepak

\begin{tabular}{|c|c|c|c|}
\hline Criterion & Range & Mean (SD) & Comment \\
\hline Incision size (mm) & $3.0-3.6$ & $3.19(0.12)$ & Mostly related to individual surgical technique \\
\hline Duration of surgical procedure from incision (minutes) & $7-45$ & $17.9(7.0)$ & Long times related to combined procedures \\
\hline Intraoperative lens (IOL) power used (D) & +14.0 to +26.0 & $+21.18(2.07)$ & Distribution identical to sales (see Figure 3) \\
\hline \multicolumn{4}{|l|}{ Ophtalmic viscosurgical device (OVD) } \\
\hline Two One (LCA) & 218 patients $(73 \%)$ & & No difference related to OVD was reported in the \\
\hline DuoVisc (Alcon) & 49 patients $(17 \%)$ & & study in terms of cataract surgery, IOL injection and \\
\hline Protea (LCA) & 29 patients $(10 \%)$ & & post-operative results \\
\hline Amvisc+ (B \& L) & 1 patient & & \\
\hline
\end{tabular}

Figure 7: Comparison Follow-ups of GENIUM AFI 5.5 Prepak and SN 60 on the Same Patient

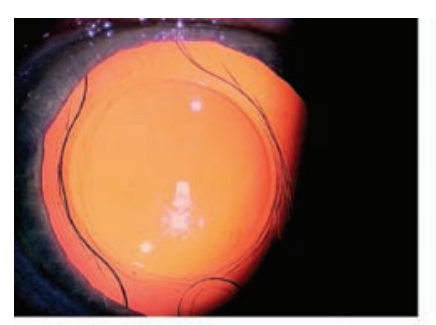

A GENIUM AFI 5.5 at 7 days

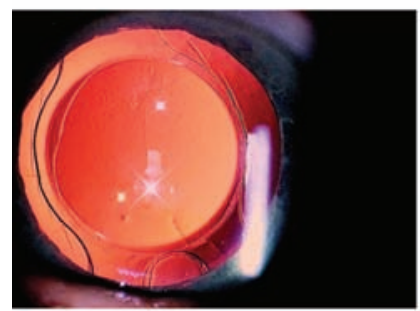

C GENIUM AFI 5.5 at 6 months

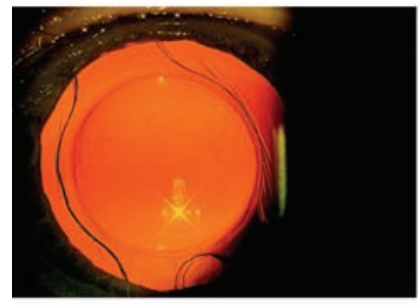

E GENIUM AFI 5.5 at 1 year

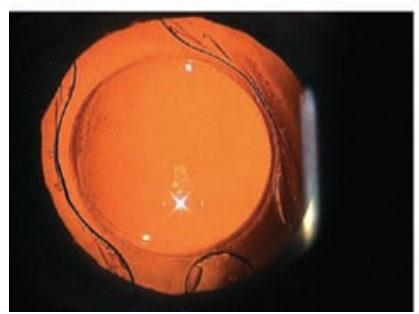

G GENIUM AFI 5.5 at 2 years

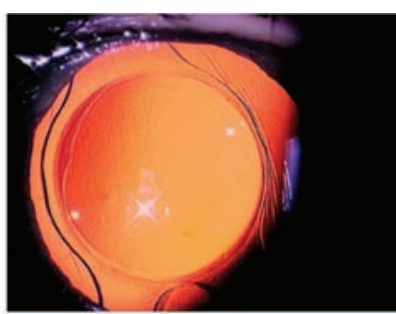

B GENIUM AFI 5.5 at 3 months

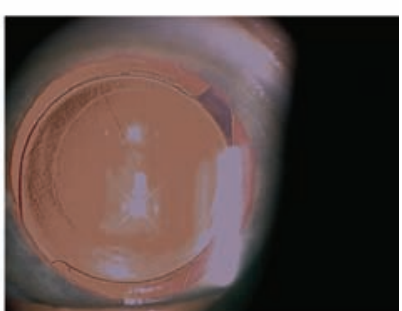

D SA 60 at 6 months

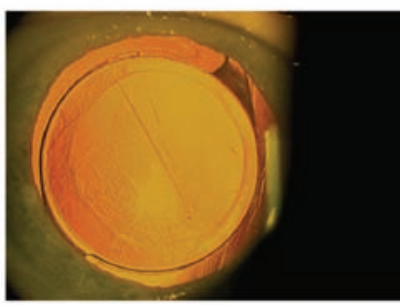

F SA 60 at 1 year

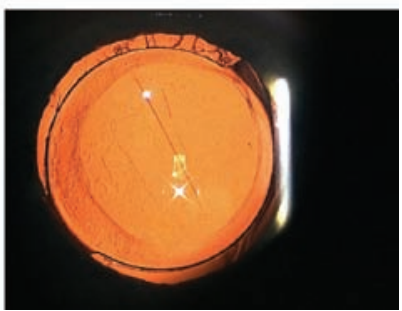

H SA 60 at 2 years

Photographs by Dr Thierry Amzallag, Institut Ophtalmologique SEMAIN.

investigators than for the whole group, confirming that surgeons should use their own statistics and use only their personal A-constant. A comparison of intraocular pressure (IOP) pre-operatively and two months post-operatively does not reveal any significant difference, as shown in Figure 6. Only one glaucomatous patient (not represented in the figure) had an increased IOP: from 24 to $27 \mathrm{mmHg}$. No correlation between IOP and use of any specific OVD has been established. Also, no cases of abnormal excessive IOP were recorded within 24 hours following surgery. No significant variation was noticed between preand post-operative IOP at two months.

Table 2: Comparison of Pre-operative Data and Post-operative Results with the GENIUM AFI 5.5 Prepak

\begin{tabular}{|c|c|c|c|}
\hline Criterion & Pre-operative & Post-operative & Comment \\
\hline Visual acuity & $0.35(0.18)$ & $0.85(0.19)$ & Largely improved with \\
\hline$(20 / 20=1.0)$ & from $0.0^{*}$ to 0.8 & from 0.3 to 1.0 & IOL (see Figure 4) \\
\hline \multicolumn{4}{|l|}{ Keratometry } \\
\hline $\mathrm{K} 1(\mathrm{~mm})$ & $7.74(0.29)$ & $7.72(0.27)$ & Unchanged \\
\hline \multirow[t]{2}{*}{$\mathrm{K} 2(\mathrm{~mm})$} & $7.65(0.27)$ & $7.65(0.28)$ & \multirow[t]{2}{*}{ Unchanged } \\
\hline & from 6.76 to 8.73 & from 6.79 to 8.62 & \\
\hline $\mathrm{Km}(\mathrm{D})$ & $43.72(1.50)$ & $43.76(1.47)$ & Unchanged \\
\hline Axial length & $23.13(0.99)$ & NA & Considered as \\
\hline (ultrasound) & from 20.40 to 26.40 & & unchanged \\
\hline \multicolumn{4}{|l|}{ Visual correction } \\
\hline Sphere (D) & $-0.15(0.39)^{* *}$ & $-0.34(0.82)$ & Difference -0.56 \\
\hline Cylinder (D) & NA & $-0.89(0.69)$ & (0.73) from target \\
\hline \multirow[t]{2}{*}{ A-constant (D) } & $118.3^{* * *}$ & $117.55(1.48)$ & Re-calculated from \\
\hline & & & SRK II (see Figure 5) \\
\hline Intraocular & $14.9(2.6)$ & $14.5(2.2)$ & Unchanged \\
\hline pressure $(\mathrm{mmHg})$ & from 10 to 24 & from 8 to 27 & (see Figure 6) \\
\hline
\end{tabular}

Quantitative results are given as mean (with standard deviation [SD] inside parentheses).

*Visual acuity non-measurable (patient just counted fingers).

**Target for post-operative refraction (emmetropia considered when data not documented). ***Estimated A-constant initially proposed by LCA; it was later modified to 118.0 (ultrasound biometry).

Table 3: Assessment of the GENIUM AFI 5.5 Prepak by Investigator

\begin{tabular}{lllll}
$\begin{array}{l}\text { Assessment by } \\
\text { Investigator }\end{array}$ & $\begin{array}{l}\text { Highly } \\
\text { Satisfactory (\%) }\end{array}$ & $\begin{array}{l}\text { Satisfactory } \\
(\%)\end{array}$ & $\begin{array}{l}\text { Medium } \\
(\%)\end{array}$ & $\begin{array}{l}\text { Poor } \\
(\%)\end{array}$ \\
\hline $\begin{array}{l}\text { Improvement of } \\
\text { visual acuity }\end{array}$ & 74 & 24 & 2 & 0 \\
\hline $\begin{array}{l}\text { Ease of use of } \\
\text { GENIUM }\end{array}$ & 38 & 30 & 27 & 6 \\
\hline $\begin{array}{l}\text { Ability to prevent } \\
\text { endophthalmitis }\end{array}$ & 64 & 36 & 0 & 0 \\
\hline
\end{tabular}

Adverse Events

There were only four adverse events during surgery: one case each of capsule rupture, capsule micro-rupture, zonula de-insertion and torn IOL (immediately replaced by an identical IOL at the same power). Other observations during surgery were: two cases of the IOL being trapped in the injector; two cases where there was difficulty with release/unfolding of the haptics; two cases where the IOL was considered 'mobile' on day zero (both became stable later); one case of mild deformation of the pupil; and five cases of punctual white deposits on the surface of the lens. These deposits are reported to stick strongly to the $\mathrm{IOL}$ and are therefore difficult to remove by aspiration. However, they neither affect patient vision nor induce reaction and they have all disappeared spontaneously within three months following surgery. 

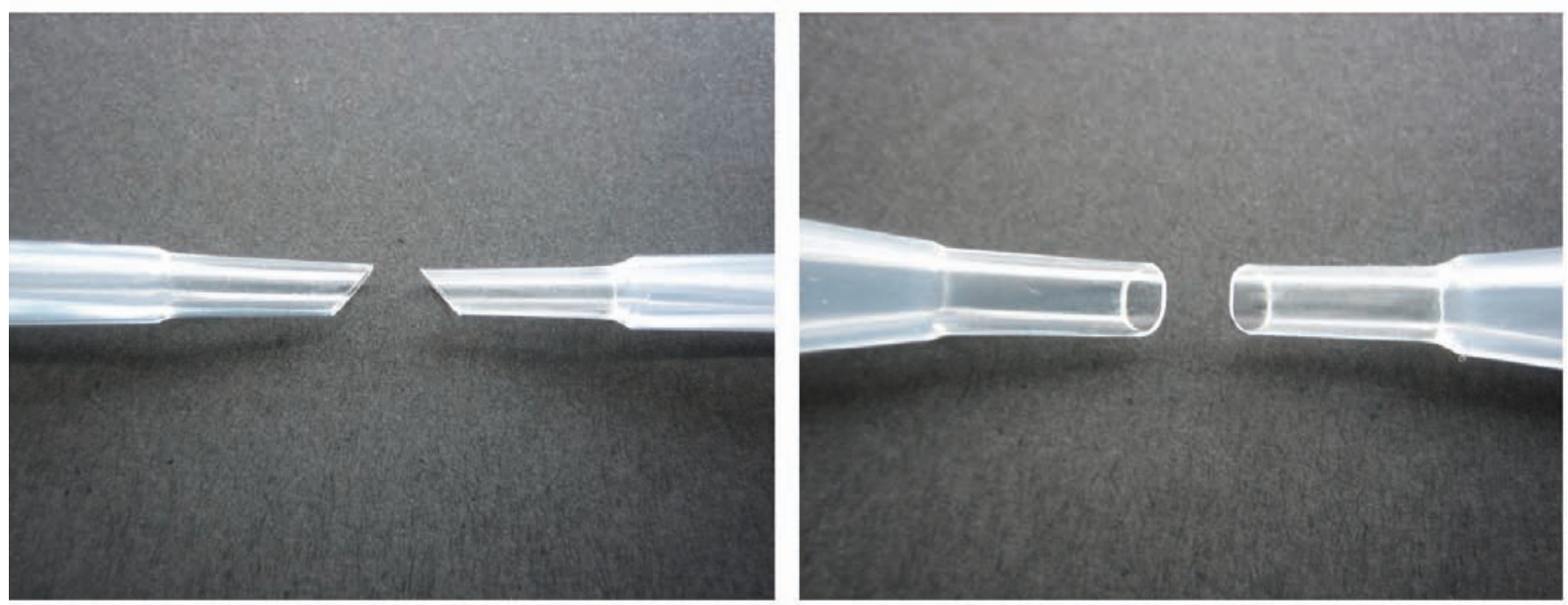

Early model (left) and new model (right): new model is present on any GENIUM injector made since mid-2006.

\section{Assessment by Investigator}

The results of the assessment of overall therapeutic efficacy by the investigator are shown in Table 3.

\section{Discussion}

The clinical results obtained with the GENIUM AFI 5.5 Prepak are excellent: improvement in VA is largely significant. No major peri- or postoperative adverse effects were reported. The majority of investigators were highly satisfied and/or satisfied with the therapeutic efficacy of the GENIUM AFI 5.5 Prepak, with 98\% reporting improvement in VA, 94\% reporting ease of use of the device and $100 \%$ reporting its ability to prevent endophthalmitis. A first minimal correction has been made to the estimated A-constant, lowering it to $118.0 \mathrm{D}$.

\section{Long-term Follow-up}

Not even one adverse event following implantation of the GENIUM AFI 5.5 Prepak has been reported to the Ministry of Health from its very first presentation at the French Society of Ophthalmology (SFO) convention in Paris in May 2004 to the time of writing (August 2008). The usual questions about the rate of posterior capsule opacification (PCO) with the GENIUM AFI 5.5 Prepak can be answered only with the fact that none of the foldable IOLs made by LCA from the same hydrophilic material over a preiod of more than 10 years is significantly different from the reference hydrophobic material. To illustrate this, Figure 7 shows photographs over a two-year period (seven days, three months, six months, one year and two years) of one eye implanted with a GENIUM AFI 5.5 Prepak; the second eye of the same patient was implanted a few days later with a hydrophobic SA 60 lens (Alcon) by the same surgeon using the same cataract extraction technique in the same environment. Photographs of the SA 60 at six months, one year and two years are provided for comparison. At two years there is no visible cell proliferation on the posterior capsule behind the GENIUM AFI 5.5 Prepak: edge contact with the capsule is continuous for both optic and haptics. Tension in the capsule is permanently maintained (as proved by folds outside the optic). The result is a stable and well centred IOL, providing good vision to the patient. From these photographs it is not possible to conclude whether there is any superiority of the hydrophobic material used by Alcon over the hydrophilic material used by LCA (istacryl and genium, respectively), in terms of the risk of PCO.

\section{Evolution of the GENIUM AFI Prepak}

Since the initial introduction of the Genium AFI Prepak onto the market in 2004, several important improvements have been made to the device to meet recent requests in eye surgery. A new $\mathrm{IOL}$ with a $6.0 \mathrm{~mm}$ diameter (GENIUM AFI 6.0 Prepak) has been developed to complete the range (the AFI 5.5 is being continued).

Also, a new tip design for the injector with smaller inside and outside diameters and a thinner wall has been introduced to provide a better fit with incisions measuring between 3.0 and $3.2 \mathrm{~mm}$ (see Figure 8). One issue with larger optics being injected through a smaller tip is the importance of the lubrication provided by the OVD. While the tendency is to use a cohesive OVD at this stage of the surgery, it must be pointed out that very cohesive OVDs do not easily coat the inside of the injector and therefore provide only poor (or even no) lubrication to the GENIUM injector. Therefore, after successful experiments with various OVDs, it has been decided that using an OVD developed by LCA was the best guarantee for successful injections using the GENIUM AFI 6.0 Prepak. A specific pack was therefore arranged with the TWO ONE OVD, called OVD Prepak.

A final improvement is that in 2007 the polypropylene used to make the injector syringes was changed to a different pharmaceutical grade. The new polypropylene material does not release its additive lubricating agent a long time after sterilisation, as the first material did. This agent seemed to be responsible for the white deposits sometimes seen on IOLs. Their chemical nature is not confirmed, but we believe they could be fat acid derivates, such as calcium stearate, used for processing polypropylene.
1. Amzallag T, Panorama des injecteurs, Visions Internationales, 1998:86:11-16.

2. Amzallag T, Injecteurs 2006: le tournant du «prêt à injecter», Flash Implants, 2005-2006:16-23.
3. Amzallag T, Injecteurs 200 : ergonomie et MICSité, Flash Implants, 2006-2007:20-30.

4. Ghipponi JP, Evolution des injecteurs, Réflexions Ophtalmologiques, 2004;9(73):20-22
5. Ghipponi JP, Evolution des injecteurs d'implants intra-oculaires, Flash Implants, 2004-2005:11-14. 


\section{Health Expenditure Determines Cataract Surgery Waiting Times}

- Delays in surgical intervention for cataracts often result in complications and poor surgical outcomes, leading the Survey of Health, Ageing and Retirement in Europe (SHARE) to investigate waiting times for cataract surgeries across 10 European countries (Austria, Germany, Sweden, The Netherlands, Spain, Italy, France, Denmark, Greece and Switzerland).

- Dr Daniel S Mojon and Dr Stefania Mojon-Azzi analysed the data alongside indicators from the Organisation for Economic Co-operation and Development (OECD) (total expenditure on health as a percentage of gross domestic product [GDP], public expenditure on health as a percentage of total health expenditure, number of acute care beds per 1,000 population and number of practising physicians per 1,000 population)

- Waiting times differed significantly between the countries analysed, and is greatly influenced by total expenditure on health, but not by other country-specific health indicators.

- Switzerland achieved the shortest waiting times (mean 1.3 months, SD 0.9). The two longest waiting times were found in Spain (mean 6.2 months, SD 6.4) and Sweden (mean 5.8 months, SD 7.2).
- The short waiting times in Switzerland and France were attributable to the high number of cataract surgeons, whereas Sweden suffers a paucity of surgeons.

- In Sweden, Italy and Switzerland, complicated cases are prioritised as more urgent than in The Netherlands, France, Germany and Spain, where complicated cases require access to beds in hospitals, which can result in delays.

\section{Cataract Surgery Waiting Times, Selected European Countries}

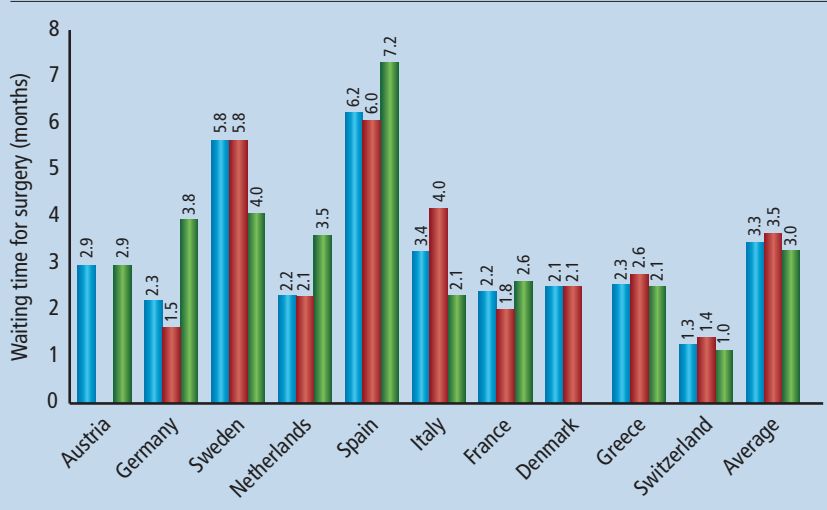

II All cataract surgery $\quad$ O Outpatient cataract surgery II Inpatient cataract surgery

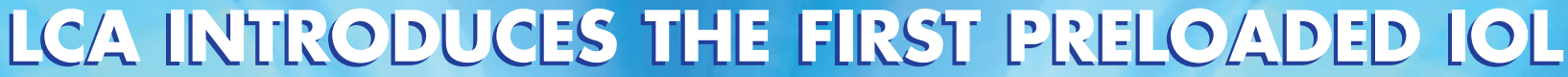

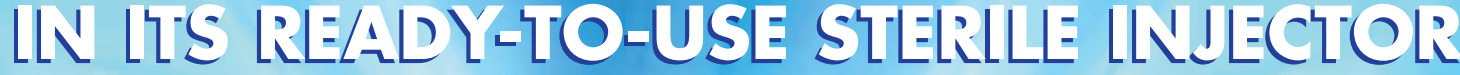

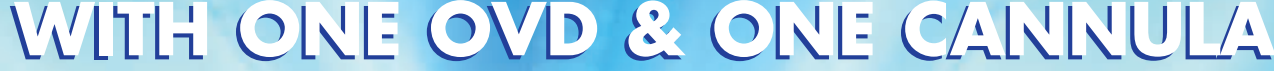
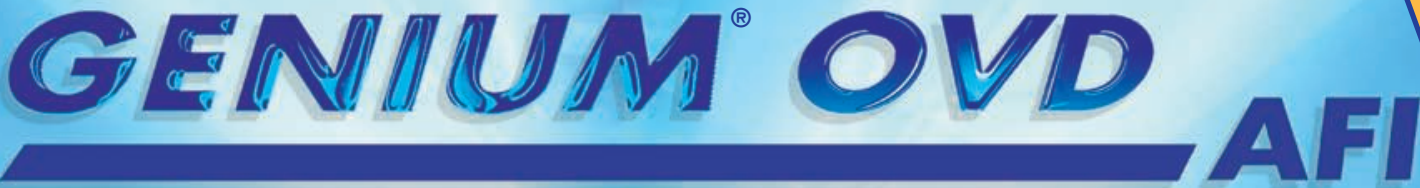
PREPAK

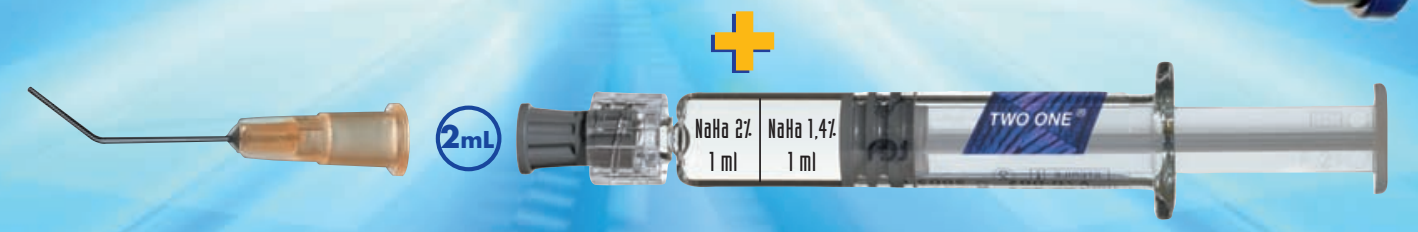

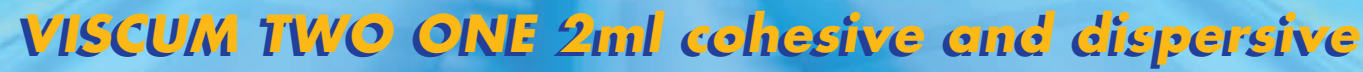

